\title{
A produção do conhecimento na área de administração de serviços de Enfermagem do programa de pós-graduação
}

\author{
THE PRODUCTION OF KNOWLEDGE IN THE FIELD OF MANAGEMENT OF NURSING SERVICES \\ IN THE GRADUATE PROGRAM IN NURSING
}

\author{
LAPRODUCCIÓN DEL CONOCIMIENTO EN EL ÁREA DE ADMINISTRACIÓN DE SERVICIOS DE \\ ENFERMERÍA DEL PROGRAMA DE POSTGRADO
}

\author{
Maria Helena Trench Ciampone ${ }^{1}$, Vanda Elisa Andres Felli², Valéria Castilho ${ }^{3}$, Paulina Kurcgant ${ }^{4}$
}

\section{RESUMO}

O presente estudo teve como objetivo identificar e analisar a produção do conhecimento na área de concentração Administração de Serviços de Enfermagem, no Curso de Mestrado e Doutorado da EEUSP. Trata-se de um estudo exploratório, descritivo, retrospectivo, pautado na análise documental. A população foi constituída por 128 produções científicas, sendo 102 (79,7\%) dissertações de mestrado e 26 (20,3\%) teses de doutorado produzidas no período de 1977 a março de 2004. Esses estudos foram analisados de acordo com o agrupamento dos temas, nas categorias propostas por Castro e Ciampone (2002). Os resultados permitiram visualizar a distribuição da produção por temática, década, método e correntes de pensamento adotadas nesses estudos. Permitiram, também, traçar considerações a respeito da trajetória, necessidades e perspectivas das pesquisas em Gerenciamento em Enfermagem no âmbito do Programa.

\section{DESCRITORES}

Educação de pós-graduação em enfermagem.

Pesquisa em administração de enfermagem.

\begin{abstract}
The study at hand had as an objective the identification and analysis of the production of knowledge in the field of Management of Nursing Services, in the Master and Doctorate Courses of the Graduate Program at the Nursing School of the University of São Paulo. It is an exploratory, descriptive, retrospective and documentary study. The population was constituted of 128 scientific productions, namely, 102 (79.7\%) master dissertations and 26 (20.3\%) doctorate theses produced between 1977 and March 2004. These works were analyzed according to theme groups in the proposed categories. Results allowed us to visualize the distribution of that production in terms of theme, decade, method and lines of thought adopted in such works. Results also allowed us to make considerations as to the trajectories, needs and perspectives of research in Nursing Management within the Program.
\end{abstract}

\section{KEY WORDS}

Education, nursing, graduate. Nursing administration research.

\section{RESUMEN}

En el presente estudio se tuvo como objetivos identificar y analizar la producción del conocimiento en el área de Administración de Servicios de Enfermería, en el Curso de Maestría y Doctorado del Programa de PostGrado de la Escuela de Enfermería de la USP (EEUSP). Se trata de un estudio exploratorio, descriptivo, retrospectivo y documental. La población estuvo constituida por 128 producciones científicas, siendo 102 (79,7\%) tesis de maestría y 26 (20,3\%) tesis de doctorado producidas en el período de 1977 a marzo del 2004. Estos estudios fueron analizados de acuerdo al agrupamiento de temas, en las categorías propuestas. Los resultados permitieron visualizar la distribución de la producción por temática; década; método y corrientes de pensamiento adoptadas en esos estudios. Permitieron, también, trazar consideraciones sobre la trayectoria, necesidades y perspectivas de las investigaciones en Gerencia en Enfermería en el ámbito del Programa.

\section{DESCRIPTORES}

Educación de postgrado en enfermería.

Investigación en administración de enfermería
1 Enfermeira. Professora Associada do Departamento de Orientação Profissional da Escola de Enfermagem da Universidade de São Paulo (EEUSP) mhciamp@usp.br

2 Enfermeira. Professoras Associada do Departamento de Orientação Profissional da EEUSP

3 Enfermeira. Professora Doutora do Departamento de Orientação Profissional da EEUSP.

4 Enfermeira. Professora Titular do Departamento de Orientação Profissional da EEUSP. 
Maria Helena T. Ciampone Vanda Elisa Andres Felli Valéria Castilho Paulina Kurcgant

\section{INTRODUÇÃO}

Devido à ausência de informações que registrem a criação e evolução da área de concentração Administração de Serviços de Enfermagem, do Programa de Pós-Graduação da Escola de Enfermagem da USP - PPGEE, torna-se necessário, para o resgate e análise da produção do conhecimento gerado nessa área, recuperar e registrar como se deu esse momento histórico acadêmico. Vale ressaltar que nesse resgate, faz-se necessário explicar como ocorreu a capacitação dos docentes uma vez que são, esses docentes, os sujeitos e agentes produtores desse conhecimento.

O resgate da história do PPGE da Escola de Enfermagem da USP (EEUSP) permite recuperar que esta foi uma das precursoras do processo de formação de enfermeiros, no âmbito de Pós-Graduação, no País, complementando informações para o entendimento de como se deu esse processo em âmbito nacional ${ }^{(1)}$.

A implantação do PPGE iniciou-se com o Curso de Mestrado, em 1973, com a área de concentração de Fundamentos de Enfermagem. Em 1974, a área de concentração de Administração de Serviços de Enfermagem foi iniciada, junto ao Departamento de Orientação Profissional. Isso ocorreu, juntamente, com as áreas de Enfermagem Pediátrica, Enfermagem Obstétrica e Neonatal e Enfermagem Psiquiátrica ${ }^{(1)}$.

A implantação da área de concentração "Administração de Serviços de Enfermagem” do Programa de Pós-Graduação da EEUSP foi feita com dificuldades, possibilidades e perspectivas em relação à capacitação dos enfermeiros, que foram comuns às outras áreas e aos outros Programas de Pós-Graduação no País ${ }^{(1)}$.

Essa área foi pioneira e única por muitos anos, no País, na capacitação de enfermeiros em Administração. Posteriormente, outro Programa, fora do estado de São Paulo, passou a oferecer a capacitação nessa área de concentração, na Universidade Federal da Bahia - UFBA ${ }^{(2)}$. Assim, atualmente, dos 25 cursos existentes, somente dois têm essa área de concentração $0^{(2)}$.

Um ponto essencial que merece ser ressaltado para a efetivação da proposta do Programa de Mestrado da Escola de Enfermagem da USP, foi o esforço dispendido por algumas docentes para a obtenção do titulo de Doutor, nos anos de 1971 a 1973, preenchendo um dos requisitos essenciais para a aprovação de Programas de Mestrado. Assim, dentre estas docentes que obtiveram o título de doutor, encontrava-se a Professora Circe de Melo
Ribeiro, que desenvolvia atividades de ensino de graduação na área de Administração em Enfermagem. Esta professora tomou para si a responsabilidade de ser precursora na capacitação de enfermeiras, no âmbito da Pós-Graduação, na especificidade das temáticas inerentes ao gerenciamento em Enfermagem.

A proposta dessa área de concentração continha disciplinas obrigatórias e optativas, sendo exigido o cumprimento de um total de 48 créditos para o Mestrando. Quanto à grade curricular, a área de concentração oferecia disciplinas obrigatórias, denominadas Administração de Serviços de Enfermagem I, II e III, ministradas em três semestres consecutivos.

A primeira turma titulada, nessa área de concentração, nos anos de 1977 a 1979, foi constituída de seis enfermeiras, sendo cinco delas docentes de diferentes Departamentos da EE e uma enfermeira chefe de serviço de um hospital do Município de São Paulo. Estas foram orientadas por seis docentes doutoras da Escola, sendo uma orientada pela docente responsável pela área de concentração em Administração - a Professora Paulina Kurcgant.

Na década de 80, nessa área de concentração, foram defendidas 23 dissertações, sendo nove orientadas por duas docentes a ela pertencentes. As demais mestrandas continuaram sendo orientadas por outras docentes da Escola e de outras áreas de concentração. Nesse momento, o mestrado tinha a explícita missão de formar docentes. Assim, vale ressaltar que, dentre as 23 Mestres tituladas, seis eram docentes da disciplina de Administração no Curso de Graduação da EEUSP.

Ainda nessa década, a Professora Paulina obteve a titulação como Doutora e passou a fazer parceria com a Dra. Circe na orientação dos alunos da área de concentração. Portanto, as seis docentes tituladas foram orientadas por elas. Essa titulação possibilitou que docentes de Administração prosseguirem na carreira acadêmica, com capacitação na área específica de atuação. A titulação seqüencial do corpo de docente, como Doutoras, propiciou a absorção de maior número de mestrandas no Programa, que passaram a ser orientadas pelas docentes da área estudada.

Nesse primeiro momento, observa-se a prioridade na titulação de professores, independente da área de conhecimento, uma vez que a missão do Mestrado era, e continua sendo, a capacitação para o exercício da docência. A seguir, outros requisitos começam a delinear-se, quando a CAPES começa a estabelecer critérios de avaliação dos Programas 
de Pós-Graduação, sendo um deles o tempo de titulação.

Dessa forma, ainda na década de 80, a avaliação do PPGEE pela CAPES demonstrou que o tempo médio de titulação oscilava entre 31 e 86 meses, enquanto os critérios adotados na época deveriam estar entre 36 a 48 meses. Assim, muitos ajustes foram feitos no programa e na área, buscando adequá-lo aos novos critérios, incluindo dentre esses a diminuição do tempo de titulação.

Ainda no que diz respeito à capacitação e titulação do corpo docente, em 1989, teve início o Curso de Doutorado do PPGEE, sendo que os primeiros doutores foram titulados a partir de abril de 1994. Isso possibilitou que na década de 90 fossem defendidas 30 dissertações, sendo que 21 foram orientadas por docentes da área de Administração de Serviços de Enfermagem e apenas nove das mestrandas foram orientadas por docentes de outros Departamentos da Escola.

Com a reformulação do PPGEE, mediante a definição de novas diretrizes da CAPES para reduzir o tempo de titulação, as disciplinas obrigatórias da área de concentração passaram a ser duas - Administração de Serviços de Enfermagem I e II ministradas em dois semestres consecutivos. Com essas novas diretrizes, o número de créditos exigidos nos Programas de Mestrado foi reduzido para 30.

Na década de 1994 a 2004, no Curso de Doutorado do Programa de Pós-Graduação EEUUP, foram defendidas 26 teses, orientadas por oito docentes da área de Administração de Serviços de Enfermagem. Nessa trajetória entre 2000 e 2004 foram defendidas 43 dissertações de mestrado, todas orientadas pelas 10 docentes da área.

Ainda na década de 90, a avaliação dos Programas de Pós-Graduação, pela CAPES, estabeleceu também, como requisito importante, a consonância entre as disciplinas ofertadas nos Programas, as linhas de pesquisa às quais deveriam vincular-se os projetos de pesquisa dos orientadores, com seus respectivos subprojetos dos Mestrandos e Doutorandos e a produção científica dos docentes e discentes pertencentes aos programas e respectivas áreas.

A partir desse fato, as linhas de pesquisa, que eram em grande número agregando temáticas pertinentes a todas as áreas de concentração, passam a ser realinhadas de acordo com as temáticas próprias das áreas de concentração. No âmbito da EEUSP, as linhas de pesquisa foram reavaliadas e reformuladas sob esse novo enfoque e na área de concentração de Administração de Serviços de En- fermagem ficaram definidas as seguintes: "Políticas e Práticas de Educação e Enfermagem”, “Gerenciamento de Recursos Humanos em Saúde e em Enfermagem" e "Gerenciamento de Ações e de Serviços de Saúde e de Enfermagem”.

Simultaneamente às reavaliações do programa, foi ocorrendo a capacitação docente, tendo todas as docentes a oportunidade de obter a titulação de Mestre e Doutor. A maioria do grupo obteve o título de doutor na área de Enfermagem e as demais nas áreas de Educação, Psicologia e Saúde Pública. Esse processo permitiu o adensando da massa crítica docente da Área de concentração de Administração de Serviços de Enfermagem no Programa, e conseqüente incremento da produção do conhecimento.

Atualmente, o Departamento no qual esta inserida esta Área de Concentração, conta com 17 docentes, sendo duas Professoras Titulares, quatro Livre-Docentes, 11 Doutores. Desse total, 13 docentes estão credenciadas para orientar no âmbito do Mestrado e dentre esses, nove para orientação de Doutorado. Até junho do ano de 2004, tivemos a formação de 102 Mestres e 26 Doutores vinculados a essa área de conhecimento.

A Área de Concentração acompanhou, nessa última década, o processo de reformulação do PPGEE que redefiniu o número de créditos para o Mestrado correspondendo a 96, sendo 30 créditos em disciplinas e 66 para a dissertação. Para o Doutorado, correspondendo a 192, sendo 28 créditos em disciplinas e 134 créditos para a tese. Outra alteração foi a redução do tempo de titulação, segundo as diretrizes da CAPES, para 30 meses no Mestrado e 48 meses no Doutorado.

A meta a ser alcançada pelo PPGEE e, conseqüentemente, pela Área de Concentração Administração de Serviços de Enfermagem, é de 27 meses para o Mestrado e de 48 meses para o Doutorado. Outras preocupações da Área são: vincular os projetos de pesquisa às linhas de pesquisa e à produção científica do corpo docente e discente; a outra é manter a relação de, no máximo, cinco orientandos por orientador.

Está tramitando uma proposta de expansão da Área de Concentração do Programa para o Doutorado, melhor articulando conteúdos das diferentes disciplinas, no sentido de conferir-lhe, internamente, mais consistência e coerência e articulação entre objetivos, disciplinas, linhas de pesquisa e projetos com a produção derivada. Assim, foi proposta a criação de novas disciplinas e o credenciamento de novos docentes titulados, com o intuito de atender
A produção do conhecimento na área de administração de serviços de enfermagem do programa de pós-graduação 
Maria Helena T. Ciampone Vanda Elisa Andres Felli Valéria Castilho Paulina Kurcgant à demanda crescente nesta área específica de pósgraduação, que passou a denominar-se "Administração de Serviços de Saúde”. A área oferece 14 disciplinas que fundamentam as temáticas desenvolvidas para orientação de mestres e doutores, com o objetivo de produzir conhecimentos relativos ao gerenciamento em enfermagem, considerando as dimensões ético-histórica, teórica e metodológica.

Nesse contexto, espera-se que a produção científica gerada nessa área de Pós-graduação subsidie a formação de competências em gerenciamento, em consonância com as atuais políticas de saúde e de ensino no País.

Entretanto, essa produção ainda não está resgatada de forma sistematizada de modo a permitir a identificação de fortalezas e fragilidades nessa área do conhecimento, bem como as lacunas a serem preenchidas por novas investigações. Aliado a este fato, o ano comemorativo dos 30 anos do Programa de Pós-Graduação da EEUSP (2003-2004) foi considerado propício para, nesse resgate histórico que explicita os elementos constitutivos da trajetória da área de Concentração Administração de Serviços de Enfermagem, realizar a presente investigação.

\section{OBJETIVO}

- Identificar e analisar a produção do conhecimento na área de concentração "Administração de Serviços de Enfermagem”, no Curso de Mestrado o Doutorado do Programa de Pós-Graduação da EEUSP.

\section{MÉTODO}

Este é um estudo exploratório, descritivo, retrospectivo e documental, com abordagem quantitativa.

A população foi constituída por 128 produções científicas, sendo 102 (79,7\%) dissertações de mestrado e 26 (20,3\%) teses de doutorado, produzidas no período de 1977 à março de 2004, na Área Concentração Administração de Serviços de Enfermagem, do Programa de Pós-graduação da Escola de Enfermagem da Universidade de São Paulo (PPGEEUSP).

A fonte para o levantamento dos dados referentes ao ano de produção, nome do autor e título da pesquisa, foram os registros da Secretária de PósGraduação da Escola de Enfermagem da EEUSP. Esses dados foram coletados em junho de 2004. Mediante a listagem elaborada pela Secretaria foram levantados, em julho de 2004, os resumos das pesquisas nos Catálogos "Informações sobre Pes- quisas e Pesquisadores em Enfermagem”, do Centro de Estudos e Pesquisas em Enfermagem da ABEn Nacional (anos de 1979-2000, 2001, 2002 e 2003) ${ }^{(3-6)}$ e no acervo de teses e dissertações da Biblioteca da EEUSP. Nesses resumos, foram buscados os dados sobre o tema do estudo, método adotado, linha de pensamento e se o estudo consistia em pesquisa cujo delineamento avançava ou não para intervenção.

A classificação dos temas segundo a linha de pensamento foi fundamentada em Minayo ${ }^{(7)}$.

Para agrupar as temáticas das produções na área da Administração em Enfermagem seguiu-se as categorias propostas ${ }^{(8)}$ : ensino de enfermagem; ensino de administração em enfermagem;; assistência de enfermagem; gerenciamento da assistência; organização, dinâmica e avaliação de serviços; gerenciamento de recursos humanos; gerenciamento de recursos materiais; gerenciamento da informação e gerenciamento de custos.

Foram agrupados na categoria Ensino de Enfermagem estudos que versavam sobre propostas de integração docente-assistencial, vivencial do professor de enfermagem e avaliação do processo ensino-aprendizagem que compreendeu levantamento de expectativas de alunos, competência interpessoal do professor e avaliação de disciplinas e de cursos relacionadas à conteúdo e estratégias pedagógicas.

Na categoria Ensino de Administração em Enfermagem foram inseridos estudos sobre análise de programas e desenvolvimento de competências relacionados à disciplina de administração, do curso de graduação e técnico. Nessa categoria também fizeram parte estudos sobre a informatização do ensino de administração em enfermagem.

Na categoria Assistência de Enfermagem foram agrupados os estudos sobre assistência à diferentes grupos de clientes institucionalizados ou não, propostas ou avaliações de intervenções de enfermagem, identificação de problemas de pacientes, formulação de diagnóstico, conhecimento do paciente ou familiares sobre patologia e tratamento, qualidade de vida do paciente, reprodução humana, técnicas de isolamento e terapias alternativas.

Foram incluídos, na categoria Gerenciamento da Assistência, os estudos sobre processos de formação de intervenções e protocolos para assistência de enfermagem, processos de implantação e avaliação de sistematização da assistência de enfermagem, estimativa do tempo da assistência e classificação de paciente. 
Na categoria Organização, Dinâmica e Avaliação de Serviços foram inseridas pesquisas sobre modelos gerenciais; processo de trabalho; proposta de organização de serviços, avaliação da qualidade de serviços por usuários internos e externos, relações de poder institucional; e, também, estudos sobre a ética nos serviços, tais como: autonomia do doente hospitalizado, percepção dos familiares sobre processo de doação de órgãos e ocorrências éticas com pessoal de enfermagem.

Foram agrupados na categoria gerenciamento de recursos humanos, os seguintes temas: educação continuada, avaliação de desempenho, rotatividade, supervisão, motivação, processo admissional, dimensionamento de pessoal, relacionamento interpessoal no ambiente de trabalho. levantamento e descrição de funções, inserção de profissionais no mercado de trabalho, saúde do trabalhador e qualidade de vida no trabalho.

O Gerenciamento de Materiais contou com um único trabalho sobre critérios de avaliação de material para a compra; no Gerenciamento de Custos foram incluídos os estudos sobre mensuração de custos de procedimentos e serviços e sobre o conhecimento de profissionais sobre custos de materiais. Na categoria Gerenciamento da Informação foram agrupadas as pesquisas sobre comunicação, elaboração e avaliação de instrumentos assistenciais e gerenciais e informatização de atividades assistenciais e gerenciais.

Os procedimentos seguidos foram os seguintes: leitura analítica dos resumos e lançamento dos dados em uma planilha no excel, onde constavam: número do estudo, tipo de produção (mestrado ou tese de doutorado), a categoria temática (segundo os critérios mencionados anteriormente), o tipo de estudo e a linha de pensamento. Os estudos que não continham esses dados no resumo foram resgatados para consulta.

Para a realização da análise quantitativa, os dados introduzidos em planilha foram sistematizados, segundo freqüências absolutas e relativas e, posteriormente, discutidos. Os resultados referentes as dissertações de mestrado e teses de doutorado foram apresentados em conjunto para facilitar a visualização e análise.

\section{RESULTADOS E DISCUSSÃO}

Os dados demonstrados na Tabela 1 permitem verificar que foram produzidas, de 1974 até março de 2004, 102 dissertações de mestrado e 26 teses de doutorado.

Tabela 1- Distribuição numérica das dissertações e teses produzidas no PPGEEUSP, área de concentração Administração de serviço de enfermagem, de 1974 a 2004 - São Paulo, 2004.

\begin{tabular}{lrrrc}
\hline & \multicolumn{2}{c}{ Mestrado } & \multicolumn{2}{c}{ Doutorado } \\
\hline Década & N & $\%$ & N & $\%$ \\
\hline $1970^{*}$ & 6 & 5,9 & - & - \\
1980 & 23 & 22,5 & - & - \\
1990 & 30 & 29,4 & 13 & 50,0 \\
$2000^{* *}$ & 43 & 42,2 & 13 & 50,0 \\
\hline Total & 102 & 100 & 26 & 100 \\
\hline
\end{tabular}

* Em 1974 foi produzida a primeira monografia.

** Foram incluídas as dissertações e teses produzidas até março de 2004.

Há um aumento significativo e crescente no número de teses e dissertações produzidas ao longo das décadas. Em relação às dissertações, a produção de 4 anos e 2 meses da década de 2000 representa $72,8 \%$ da soma das produções nas décadas anteriores. O número de teses produzidas nesse período é igual à produção de toda década de 1990. Salienta-se que o Curso de Doutorado foi iniciado em 1989, titulando o primeiro doutor a partir de 1994. Observa-se o aumento gradativo do número de titulações, segundo as décadas, que acompanha o incremento da pós-graduação no País.

Os temas abordados nas dissertações e teses, segundo as diferentes décadas, podem ser observados na Tabela 2.
A produção do conhecimento na área de administração de serviços de enfermagem do programa de pós-graduação 
Maria Helena T. Ciampone Vanda Elisa Andres Felli Valéria Castilho Paulina Kurcgant

Tabela 2 - Distribuição das dissertações e teses, produzidas no PPGEEUSP, área de concentração Administração de serviço de enfermagem, de 1974 a 2004, segundo as categorias temáticas - São Paulo, 2004.

\begin{tabular}{|c|c|c|c|c|c|}
\hline \multirow[b]{2}{*}{ Década } & \multirow[b]{2}{*}{ Tema } & \multicolumn{2}{|c|}{ Mestrado } & \multicolumn{2}{|c|}{ Doutorado } \\
\hline & & $\mathbf{N}$ & $\%$ & $\mathbf{N}$ & $\%$ \\
\hline \multirow{4}{*}{70} & Assistência de Enfermagem & 3 & 50,2 & - & - \\
\hline & Ensino de Enfermagem & 1 & 16,6 & - & - \\
\hline & Gerenciamento de Rec. Humanos & 1 & 16,6 & - & - \\
\hline & Gerenciamento de Informação & 1 & 16,6 & - & - \\
\hline \multirow{9}{*}{80} & Subtotal & 6 & 100 & - & - \\
\hline & Assistência de Enfermagem & 9 & 39,1 & - & - \\
\hline & Gerenciamento de rec. Humanos & 5 & 21,8 & - & - \\
\hline & Org. Din e Avaliação de Serviços & 3 & 13,1 & - & - \\
\hline & Ensino de Enfermagem & 2 & 8,7 & - & - \\
\hline & Gerenciamento da Informação & 2 & 8,7 & - & - \\
\hline & Gerenciamento de Rec. Materiais & 1 & 4,3 & - & - \\
\hline & Gerenciamento da Assistência & 1 & 4,3 & - & - \\
\hline & Subtotal & 23 & 100 & - & - \\
\hline \multirow{8}{*}{90} & Gerenciamento de Rec. Humanos & 10 & 33,3 & 7 & 53,9 \\
\hline & Gerenciamento da Assistência & 5 & 16,6 & - & - \\
\hline & Org. Din e Avaliação de Serviços & 5 & 16,6 & 2 & 15,4 \\
\hline & Assistência de Enfermagem & 4 & 13,3 & 4 & 30,7 \\
\hline & Gerenciamento de Custos & 2 & 6,7 & - & - \\
\hline & Ensino de Enfermagem & 2 & 6,7 & - & - \\
\hline & Gerenciamento da Informação & 1 & 3,4 & - & - \\
\hline & Ensino de Administração em Enf. & 1 & 3,4 & - & - \\
\hline \multirow{11}{*}{00} & Subtotal & 30 & 100 & 13 & 100 \\
\hline & Gerenciamento de Rec. Humanos & 15 & 34,9 & 4 & 30,8 \\
\hline & Org. Din e Avaliação de Serviços & 8 & 18,7 & 2 & 15,4 \\
\hline & Gerenciamento de Custos & 6 & 13,9 & - & - \\
\hline & Gerenciamento da Assistência & 5 & 11,7 & - & - \\
\hline & Ensino de Enfermagem & 4 & 9,3 & 5 & 38,4 \\
\hline & Ensino de Administração em Enf. & 2 & 4,6 & 2 & 15,4 \\
\hline & Assistência de Enfermagem & 2 & 4,6 & - & - \\
\hline & Gerenciamento da Informação & 1 & 2,3 & - & - \\
\hline & Subtotal & 43 & 100 & 13 & 100 \\
\hline & Total & 102 & 100 & & 100 \\
\hline
\end{tabular}

Observa-se na Tabela 2 que os temas Ensino de Enfermagem, Assistência de Enfermagem, Gerenciamento de Recursos Humanos e Gerenciamento da Informação foram pesquisados em todas as décadas. O tema mais estudado nas quatro décadas foi Gerenciamento de Recursos Humanos com 31 produções (30,4\%), seguido do tema Assistência de Enfermagem com 18 (17,6\%), Organização, dinâmica e Avaliação de Serviços com 16 (15,8\%), Gerenciamento da Assistência com 11 (10,8\%), Ensino de Enfermagem com 9 (8,8\%), Gerenciamento de Custos com 8 (7,9\%), Gerenciamento da Informação com 5 (4,9\%), Ensino de Administração em Enfermagem com 3 (2,9\%) e Gerenciamento de recursos materiais com $1(0,9 \%)$.
Quanto aos temas das teses de doutorado o de Gerenciamento de Recursos Humanos conta com 11 produções (42,3\%), seguido do tema Ensino de Enfermagem com 5 (19,2\%), Assistência de Enfermagem e Organização, dinâmica e Avaliação de Serviços com 4 (15,4\%) cada um e Ensino de Administração em Enfermagem com 2 (7,7\%).

A construção do conhecimento em gerenciamento de serviços é relativamente recente ${ }^{(9)}$. A ciência da administração foi construída a partir de experiências no setor secundário, ou seja, nas indústrias. As autoras referem que, hoje, face o grande desenvolvimento do setor terciário (serviços), surge a necessidade de produção de conhecimento para esta nova realidade. O desenvolvimento dos 
conhecimentos em gerenciamento de serviços, dado o contexto, passa a ser de maior relevância também para a enfermagem e saúde.

A produção científica da Enfermagem brasileira, na década de 90, aponta uma forte concentração nas áreas organizacional e profissional, áreas em que se classifica a produção da Área de Concentração Ad- ministração de Serviços de Enfermagem ${ }^{(10)}$. Esse dado corrobora com a análise feita ${ }^{(11)}$ afirmando que, na década de 90, a Enfermagem brasileira começa a discutir a prática profissional como trabalho.

Os dados relativos ao método de abordagem podem ser visualizados na Tabela 3 .

Tabela 3 - Distribuição das dissertações e teses, produzidas no PPGEEUSP, área de concentração administração de serviço de enfermagem, de 1974 a 2004, segundo o método de abordagem - São Paulo, 2004.

\begin{tabular}{|c|c|c|c|c|c|}
\hline \multirow[b]{2}{*}{ Década } & \multirow[b]{2}{*}{ Método } & \multicolumn{2}{|c|}{ Mestrado } & \multicolumn{2}{|c|}{ Doutorado } \\
\hline & & $\mathbf{N}$ & $\%$ & $\mathbf{N}$ & $\%$ \\
\hline \multirow{4}{*}{70} & Quantitativo & 6 & 100 & - & - \\
\hline & Qualitativo & - & - & - & - \\
\hline & Quantitativo-Qualitativo & - & - & - & - \\
\hline & Subtotal & 6 & 100 & - & - \\
\hline \multirow{4}{*}{80} & Quantitativo & 20 & 86,9 & - & - \\
\hline & Qualitativo & 3 & 13,1 & - & - \\
\hline & Quantitativo-Qualitativo & - & - & - & - \\
\hline & Subtotal & 23 & 100 & - & - \\
\hline \multirow{4}{*}{90} & Quantitativo & 16 & 53,4 & 1 & 7,7 \\
\hline & Qualitativo & 13 & 43,3 & 12 & 92,3 \\
\hline & Quantitativo-Qualitativo & 1 & 3,3 & - & - \\
\hline & Subtotal & 30 & 100 & 13 & 100 \\
\hline \multirow{5}{*}{00} & Quantitativo & 21 & 48,9 & 2 & 15,4 \\
\hline & Qualitativo & 19 & 44,2 & 11 & 84,6 \\
\hline & Quantitativo-Qualitativo & 3 & 6,9 & - & - \\
\hline & Subtotal & 43 & 100 & 13 & 100 \\
\hline & Total & 102 & 100 & 26 & 100 \\
\hline
\end{tabular}

Em relação ao método de pesquisa adotado nas dissertações de mestrado, observa-se na Tabela 3 que houve predomínio de estudos quantitativos na década de 70 e 80 e na década de 90 um equilíbrio entre os 2 métodos. Foram produzidos 63 (61,7\%) estudos quantitativos, 35 (34,4\%) qualitativos e 4 (3,9\%) quantitativo-qualitativo. No curso de doutorado houve uma inversão, foram produzidos 23 $(88,46 \%)$ estudos qualitativos e apenas $3(11,54)$ quantitativos.
Observa-se uma tendência de desenvolvimento de estudos qualitativos em Enfermagem. Outro estudo realizado ${ }^{(12)}$ também identificou esse predomínio em pesquisas atualmente, verificando o uso desse método em quase metade delas (44,6\%); o método quanti-qualitativo em $40,3 \%$ e $12,6 \%$ o método quantitativo.

A análise relativa ao método, realizada em estudo que aborda temática específica sobre Administração em Enfermagem, como a saúde do trabalhador de enfermagem, confirma essa tendência ${ }^{(13)}$.
A produção do conhecimento na área de administração de serviços de enfermagem do programa de pós-graduação 
Maria Helena T. Ciampone Vanda Elisa Andres Felli Valéria Castilho Paulina Kurcgant

Tabela 4 - Distribuição das dissertações e teses, produzidas no PPGEEUSP, área de concentração Administração de serviço de enfermagem, de 1974 a 2004, segundo as linhas de pensamento - São Paulo, 2004.

\begin{tabular}{|c|c|c|c|c|c|}
\hline \multicolumn{2}{|l|}{ Curso } & \multicolumn{2}{|c|}{ Mestrado } & \multicolumn{2}{|c|}{ Doutorado } \\
\hline Década & Linha & $\mathrm{N}$ & $\%$ & $\mathrm{~N}$ & $\%$ \\
\hline \multirow{4}{*}{70} & Compreensiva & - & - & - & - \\
\hline & Positivista & 6 & 100 & - & - \\
\hline & Materialismo HD & - & - & - & - \\
\hline & Subtotal & 6 & 100 & - & - \\
\hline \multirow{4}{*}{80} & Compreensiva & 1 & 4,3 & - & - \\
\hline & Positivista & 20 & 87,1 & - & - \\
\hline & Materialismo HD & 2 & 8,6 & - & - \\
\hline & Subtotal & 23 & 100 & - & - \\
\hline \multirow{4}{*}{90} & Compreensiva & 14 & 46,6 & 10 & 76,9 \\
\hline & Positivista & 16 & 53,4 & 1 & 15,4 \\
\hline & Materialismo HD & - & - & 2 & 7,7 \\
\hline & Subtotal & 30 & 100 & 13 & 100 \\
\hline \multirow{5}{*}{00} & Compreensiva & 21 & 48,9 & 2 & 15,4 \\
\hline & Positivista & 19 & 44,2 & 11 & 84,6 \\
\hline & Materialismo HD & 3 & 6,9 & - & - \\
\hline & Subtotal & 43 & 100 & 13 & 100 \\
\hline & Total & 102 & 100 & 26 & 100 \\
\hline
\end{tabular}

Conforme evidenciado na Tabela 4, no curso de mestrado, a linha de pensamento mais adotada para produção de dissertações foi a positivista com 63 produções (61,7\%), a compreensiva com 35 (34,4\%) e o materialismo histórico dialético com 4 (3,9\%). No curso de doutorado foi a compreensiva com 20 produções $(76,8 \%)$ e a positivista e o materialismo histórico dialético com valores iguais de 3 (11,6\%).

Em relação à possibilidade de intervenção nas pesquisas, existem descritos três níveis de pesqui$\mathrm{sa}^{(14)}$, considerando-se, respectivamente, as do nível I - as investigações exploratório-descritivas; nível II - as correlacionais comparativas; e nível III - as experimentais. Para os autores, a pesquisa experimental pode avançar para a intervenção, sendo que as demais são restritas quanto a essa possibilidade. Das 102 dissertações, apenas 5 (4,9\%) foram de intervenção e das 26 teses de doutorado, 4 $(15,35 \%)$.

Estudo realizado sobre a temática saúde do trabalhador de enfermagem ${ }^{(13)}$, no enfoque do Gerenciamento em enfermagem, mostra a mesma tendência, em relação às linhas de pensamento e à possibilidade de intervenção. A maioria dos estudos está fundamentada no positivismo, um número menor na linha compreensiva e, uma minoria, no materialismo histórico-dialético. O fato da maioria dos estudos estarem fundamentados no positivismo dificulta a possibilidade de intervenção, uma vez que nessa linha de pensamento, a intencionalidade é identificar, descrever e $\operatorname{comparar}^{(7)}$, não fundamentando intervenções que visem a transformação da realidade.

\section{CONCLUSÃO}

Como aspectos a serem destacados com a análise da produção nessa área apreendeu-se que houve um aumento significativo do número de produção de dissertações e teses, sendo de maior expressão o período compreendido entre janeiro do ano de 2000 a março de 2004. A produção nesse período superou a produção total da década de 90 .

Quanto aos temas das pesquisas, há evidente concentração da produção nos temas vinculados ao Gerenciamento de Recursos Humanos, seguido de trabalhos pertinentes à Assistência de Enfermagem. Esta concentração justifica-se, em parte, pela problemática mais contundente, vivida pelos enfermeiros assistenciais no cotidiano dos seus processos de trabalho e, em parte, pelo fato das linhas de pesquisa até a década de 90 não focarem na área de concentração os temas próprios dessa área de conhecimento. A temática Gerenciamento de Recur- 
sos Materiais, foi a menos investigada nessas décadas, seguida pelas temáticas Ensino de Administração em Enfermagem e Gerenciamento da Informação, apontando para lacunas na produção do conhecimento nessas áreas.

Quanto ao método adotado, constatou-se que houve a predominância dos métodos quantitativos nas duas primeiras décadas de existência da área de concentração no mestrado e de métodos qualitativos no doutorado. Embora no campo das pesquisas em saúde, as abordagens qualitativas se faziam presentes nas décadas de 60 e $70^{(15)}$, na Enfermagem, passam a serem realizadas a partir da década de 90 .

Quando analisados os estudos em relação às linhas de pensamento, constatou-se que a grande maioria das dissertações de mestrado e teses de doutorado restringiu-se ao âmbito de investigações exploratórias descritivas. Apenas uma minoria desses estudos chega à intervenção. No doutorado, esse número de estudos é maior do que no mestrado, mas indicando a necessidade de serem desenvolvidos estudos que avancem para propostas de intervenção.

\section{REFERÊNCIAS}

(1) Anais do “15 anos de Pós-Graduação na Escola de Enfermagem da Universidade de São Paulo: reflexos e perspectivas”; 1988 out. 24-25; São Paulo. São Paulo: EEUSP; 1990.

(2) Brasil. Ministério da Educação. Coordenação de Aperfeiçoamento de Pessoal de Nível superior-CAPES; [on line] Brasília; 2004. Disponível em: <http:// www.capes.gov.br> (08 set. 2004)

(3) Associação Brasileira de Enfermagem. Centro de Estudos e Pesquisas em Enfermagem. Informações sobre pesquisas e pesquisadores em enfermagem -19792000.[CD ROM] Brasília: ABEn; 2000.

(4) Associação Brasileira de Enfermagem. Centro de Estudos e Pesquisas em Enfermagem. Informações sobre pesquisas e pesquisadores em enfermagem. Brasília: ABEn; 2001.

(5) Associação Brasileira de Enfermagem. Centro de Estudos e Pesquisas em Enfermagem. Informações sobre pesquisas e pesquisadores em enfermagem. Brasília: ABEn; 2002.

(6) Associação Brasileira de Enfermagem. Centro de Estudos e Pesquisas em Enfermagem. Informações sobre pesquisas e pesquisadores em enfermagem.[online] Brasília: ABEn; 2003. Disponível em < http://www.abennacional.org.br/bc.php> (15 jun. 2004)

(7) Minayo MCS. O desafio do conhecimento: pesquisa qualitativa em saúde. $2^{a}$ ed. São Paulo: HUCITEC 1993.
Finalizando, as autoras consideram que o presente estudo, apesar das limitações inerentes à pesquisa documental, contribui para o resgate e análise da produção do conhecimento na área de Administração de Serviços de Enfermagem, do Programa de Pós-Graduação da EEUSP, indicando caminhos e perspectivas para a continuidade e avanço do ensino e da pesquisa.

As fragilidades identificadas em algumas áreas temáticas mereceram propostas de disciplinas específicas, bem como de desenvolvimento de projetos de investigação, albergados nos respectivos grupos de pesquisa. Com isso, também se pretende fortalecer as linhas de pesquisa menos desenvolvidas. Um outro movimento faz-se no sentido de estimular projetos de pesquisa cujos desenhos metodológicos propiciem intervenções, face às necessidades constantes de transformação da realidade em saúde.

(8) Castro AMF, Ciampone MHT. Estudo bibliométrico das teses e dissertações sobre a temática administração em enfermagem no período de 1979 a 2000. In: Anais do $2^{\circ}$ Encontro Internacional de Pesquisa em Enfermagem; 2002 out. 28-31; Águas de Lindóia. São Paulo: Escola de Enfermagem da USP; 2002.

(9) Gutierrez MGR, Leite JL, Pagliuca LMF, Erdman AL. Os múltiplos problemas pesquisados e a pesquisar na enfermagem. Rev Bras Enferm 2002; 55(5):535-41.

(10) Prado ML, Gelbecke FL. Produção do conhecimento em enfermagem no Brasil: as temáticas de investigação. Rev Bras Enferm 2001; 54(1):34-42.

(11) Araújo MJS. A contribuição da Revista Brasileira de Enfermagem. In: Anais do $7^{\circ}$ Seminário Nacional de Pesquisa em Enfermagem; 1994; jun. 5-9; Fortaleza. Fortaleza: ABEn-Seção-CE; 1994. p. 115-28.

(12) Felli VEA, Torres VLS. Quem somos? Que caminhos trilhamos na pesquisa em enfermagem? Uma pesquisa interativa. [CD ROM]. In: Anais do 53 Congresso Brasileiro de Enfermagem; 2001 out. 914; Curitiba. Curitiba: ABEn-Seção-Pr; 2001.

(13) Felli VEA. A saúde do trabalhador e o gerenciamento em enfermagem. [livre-docência] São Paulo (SP): Escola de Enfermagem da USP; 2002.

(14) Burns N, Grove SK. The practice of nursing research: conduct critique and utilizations. Philadelphia: W. B. Sauders; 1987.

(15) Nunes ED, organizador. Medicina social: aspectos históricos e teóricos. São Paulo: Global; 1983.
A produção do conhecimento na área de administração de serviços de enfermagem do programa de pós-graduação
Correspondência: Maria Helena T. Ciampone Av. Prefeito Fábio Parada, 484 - Ap. 51

- Vila Mariana - São Paulo 04116-000 - SP

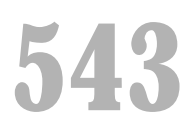

Rev Esc Enferm USP 2005; 39(Esp.):535-43. 